\title{
Mesurer l'importance de la biodiversité pour les sociétés forestières des pays du Sud. Une méthode d'investigation pluridisciplinaire
}

\author{
Manuel Boissière ${ }^{\mathrm{a}}$, Marieke Sassen ${ }^{\mathrm{b}}$ \\ a Ethnobotaniste, CIRAD, UR Ressources forestières et politiques publiques, TA 10/D, Campus international de Baillarguet, \\ 34398 Montpellier cedex 5, France \\ b Écologue, CIFOR, PO Box 6596, JKPWB Jakarta 10065, Indonésie
}

\begin{abstract}
Les conditions de production des connaissances ou d'exercice des métiers de la recherche contraignent parfois le chercheur à adopter des méthodes d'enquêtes rapides qui sont le résultat de compromis entre la demande expresse du bailleur et l'exigence théorique et méthodologique de la démarche de recherche. NSS se fait ici l'écho, au travers d'expériences singulières en Asie et en Afrique, des problèmes que ces contextes soulèvent mais aussi des réponses que les équipes inventent, et privilégie en cela l'enjeu scientifique et éthique attaché à ce nouveau mode d'exercice du travail de chercheur. Et il est intéressant de constater que la maîtrise de ces situations d'urgence passe par une posture inscrite résolument dans le point de vue de l'interdisciplinarité.
\end{abstract}

La Rédaction

\section{Mots-clés :} perceptions; paysages forestiers; priorités locales; méthodes d'investigation rapide

\begin{abstract}
Résumé - Les méthodes d'investigation rapide sont souvent critiquées par la communauté scientifique, mais il est crucial de proposer des méthodes rigoureuses, au-delà des enquêtes académiques de longue durée, permettant de répondre aux attentes des institutions de développement et des décideurs. Dans le contexte de recherches sur la biodiversité, le CIFOR (Center for International Forestry Research) a développé un jeu de méthodes permettant d'étudier l'importance des ressources et des paysages pour les communautés forestières, et les implications des perceptions locales pour la biodiversité et la conservation. Il s'inspire d'idées venant à la fois des sciences sociales (socioéconomie, anthropologie, ethnobotanique) et des sciences naturelles (écologie, botanique, pédologie). À travers des expériences de terrain en Afrique et en Asie, nous analysons comment le Multidisciplinary Landscape Assessment (MLA) peut répondre aux attentes sans céder à la superficialité des méthodes rapides classiques.
\end{abstract}

\section{Keywords:}

perceptions; forest landscapes; local priorities; rapid appraisal methods

\begin{abstract}
Assessing the importance of biodiversity for indigenous forest communities in developing countries. A multidisciplinary approach. Rapid appraisal methods are often criticized by the scientific community for their superficiality and the often contested quality of information that they provide. It is however crucial to propose rigorous methods that meet the demands of development institutions and decision makers, beyond academic and long-term survey techniques. In the context of biodiversity research, the MLA (Multidisciplinary Landscape Assessment) - a set of methods developed by CIFOR (Center for International Forestry Research) - assesses the reasons for which resources and landscapes are important for forest communities and the implications for biodiversity and conservation. It is built on both social sciences (socio-economics, anthropology, ethno-botany) and natural sciences (ecology, botany, soil science). Taking experiences in the field in Cameroon, Gabon, the Philippines, Vietnam, Kalimantan and West Papua, we analyze the ways in which MLA can meet the different expectations without falling into the traps of conventional rapid appraisal methods. We conclude that this multidisciplinary approach is a flexible diagnostic tool that can serve as a basis for more in depth and longer-term studies. In this type of study, special care should be taken regarding ethical issues as well as the local impacts of research on the longer term and the dissemination of results amongst the targeted communities.
\end{abstract}

Auteur correspondant : M. Boissière, m.boissiere@cgiar.org

M. Boissière est actuellement en affectation au CIFOR (Center for International Forestry Research) à Bogor (Indonésie). 


\section{Introduction}

Les études de la biodiversité, dont les tenants de la recherche-action se sont fait une spécialité, ont produit un savoir basé essentiellement sur des évaluations $\mathrm{d}^{\prime}$ ordre biologique des forêts tropicales ${ }^{1}$. Les méthodes de RAP (Rapid Appraisal Program), utilisées récemment en Papouasie occidentale par Conservation International (McKenna et al., 2002; Richards et Suryadi, 2002), produisent quantité d'informations sur les écosystèmes forestiers, mais le facteur humain y est un élément accessoire, en général considéré pour son impact négatif sur la biodiversité (Cyranoski, 2006). Ces études se différencient des nombreuses méthodes servant à faciliter les relations entre populations locales et projets de développement. En effet, les méthodes dites de RRA (Rapid Rural Appraisal) ou de PRA (Participatory Rural Appraisal), dont Conway et Chambers ont été les pionniers, sont légion depuis une vingtaine d'années ; elles tentent de réconcilier les priorités et les perceptions des populations rurales des pays du Sud avec les attentes et les exigences des preneurs de décisions (Conway, 1987 ; FAO,1990 ; Chambers, 1994; Holland et Blackburn, 1998 ; Selener, 1997; IFAD et al., 2001). Ces études ont pour objectif de mieux intégrer les populations dans les projets de développement censés leur bénéficier. Leurs méthodes utilisent un jeu de techniques d'enquête devant permettre de capter le point de vue local (cartographie participative, exercice de comptage, modélisation, etc.), mais elles sont largement critiquées à cause de leur usage systématique et répétitif, sans lien ni continuité entre les différentes enquêtes, et à cause de leur utilisation par des agents du développement souvent peu qualifiés. Reproche leur a souvent été fait (IFAD et al., op. cit.) de servir à légitimer et sociabiliser des projets de développement qui autrement ne seraient pas forcément acceptés par les populations locales. De plus, la pression des bailleurs de fonds limite le temps que les enquêteurs peuvent passer parmi les communautés et donc la qualité de l'information récoltée.

Depuis 1998, une équipe de chercheurs du CIFOR a développé une approche apportant des informations sur les rapports que les sociétés vivant dans ou à proximité des forêts tropicales pouvaient entretenir avec leur environnement (Multidisciplinary Landscape Assessment [MLA] / Évaluation multidisciplinaire du paysage $\left.^{2}\right)$. Ce jeu de méthodes a été développé dans

\footnotetext{
${ }^{1}$ Cet article a pour origine des études menées par le CIFOR en Indonésie et dans plusieurs autres pays tropicaux. Il fait suite à une note de lecture sur un ouvrage décrivant le jeu de méthodes, publiée dans NSS (Friedberg, 2006), et à une communication présentée au IV ${ }^{\mathrm{e}}$ Congrès international d'ethnobotanique (ICEB 2005), qui s'est tenu à Istanbul en août 2005 (Boissière et Sassen, 2005).

2 Nous utilisons dans cet article la traduction du terme anglosaxon landscape : paysage, tout en reconnaissant le manque de
}

le contexte de discussions au sein de la communauté scientifique sur le problème de la valeur locale des ressources forestières (Sheil et Wunder, 2002) et de la participation des communautés locales dans la gestion de ces ressources (Sheil et al., 2006). En effet, l'avis et les souhaits des populations locales sont souvent ignorés lors des prises de décisions concernant la gestion des ressources et des paysages forestiers.

Ces méthodes s'inscrivent clairement dans le cadre de recherches concernant la biodiversité et sa conservation, et, en ce sens, elles diffèrent de celles de RRA/PRA précédemment décrites, tout en utilisant certains de leurs outils. Les méthodes classiques d'étude de la biodiversité se basent en général sur la double question : Que trouvet-on et où ? L'étude des espèces, animales et végétales, de leur localisation et de leur dynamique est caractéristique de ce type de méthode. L'approche du CIFOR apporte trois questions supplémentaires, permettant une analyse qui prenne en compte les facteurs humains :

1. Pourquoi et pour qui les ressources naturelles sontelles importantes?

2. Cette importance est-elle quantifiable?

3. Quelles sont les menaces pour la biodiversité et pour les populations qui en dépendent?

Développées à Kalimantan Est, dans la région du bassin de la Malinau, les méthodes ont été testées et appliquées ailleurs en Indonésie (Papouasie occidentale, Sumatra) et dans d'autres pays (Vietnam, Philippines, Cameroun, Gabon, Bolivie et Mozambique $)^{3}$.

Nous souhaitons, dans cet article, débattre de l'intérêt et des difficultés liés à l'utilisation de méthodes d'investigation rapide. Ces méthodes permettent-elles de collecter rapidement et rigoureusement des informations sur les perceptions et les priorités des populations locales en termes de développement et de gestion de la biodiversité? Leur limitation dans le temps pose en effet le problème des relations que les chercheurs établissent avec les communautés locales - questions d'éthique de la recherche, du type d'informations recueillies - et, par conséquent, de la validité des résultats obtenus.

Nous apportons notre point de vue, en partageant notre expérience de l'application du MLA. Nous exposons les aspects positifs qui ont retenu notre intérêt sur les divers sites où cette méthode a été employée, mais aussi les difficultés que nous avons eues à surmonter et les questions qui se posent lorsqu'on travaille sur des projets de courte durée (rarement plus d'un mois par village), avec des populations locales.

précision du concept en français et les nombreuses interprétations dont il a fait l'objet. Nous employons ici le terme «paysage » pour désigner l'ensemble du territoire et de ses composantes (ressources, types de végétation, sites spéciaux) qu'une communauté reconnaît et s'approprie.

${ }^{3}$ Pour plus d'informations concernant les activités liées à l'usage de cette approche, voir http://www.cifor.cgiar.org/mla/ 


\section{Terrain express, une utopie?}

Nous souhaitons poser ici le problème des méthodes d'investigation rapide et de la place du chercheur sur son terrain, dans le cadre de projets de recherche touchant à des problématiques de conservation. Durant le colloque d'ethnobotanique d'Istanbul (cf. supra, note 1), la présentation du MLA a suscité une discussion sur les relations des chercheurs avec des populations considérées comme «fragiles » et «marginales » (notamment aux Philippines). Les problèmes d'éthique liés à la collecte de données (concernant, par exemple, l'usage du savoir des shamans ou le traitement des échantillons d'herbier) ont été abordés, ainsi que, d'un point de vue plus général, la validité scientifique de recherches réalisées dans un temps limité. À ce propos, Céfaï (2003, p. 557), dans son exposé sur les conditions dans lesquelles un terrain doit s'effectuer, semble s'opposer à toute méthode d'investigation rapide : "Le survol ou survey qui engrange des informations selon des procédures standardisées sans moyen de les vérifier auprès des acteurs et sans prise sur leurs contextes d'expérience et d'activité est exclu : sans liens solides avec des informateurs privilégiés, la plupart des données obtenues seront soit de complaisance, induites par les questions posées par l'enquêteur, soit anodines ou banales, puisées dans des répertoires de stéréotypes à usage public. » À partir de quel moment passe-t-on du survey, pour reprendre l'expression de Céfaï, à une véritable enquête de terrain ? Le problème ne vient pas uniquement du temps passé parmi les populations locales, mais également du positionnement du chercheur vis-à-vis de son terrain. En effet, lorsqu'il travaille dans le cadre d'une recherche finalisée, le chercheur doit faire face à des choix éthiques par rapport à des problèmes politiques ou sociaux. Choix et activités scientifiques se font dans le contexte de relations complexes, où sciences, société et politiques forment en fait des «collectifs hétérogènes » (Latour, 2001), qui sont du même type, finalement, que les systèmes observés par les anthropologues dans les sociétés qu'ils étudient : des systèmes où les rapports sociaux jouent un rôle-clé dans la construction des discours et des perceptions, et où personne n'est isolé, pas même le scientifique. Autrement dit, notre interprétation des perceptions locales sur la forêt doit être faite en tenant compte de nos propres systèmes de représentations.

Schwartz (1993) considère qu'il n'y a pas d'observation neutre qui serait sans effet sur les comportements des populations étudiées; mais, selon lui, le chercheur finit par se voir attribuer une place, une «case » par la société sur laquelle il enquête. Cette position peut certes biaiser les résultats, mais elle peut aussi agir comme révélatrice de «logiques sociales endogènes ", au travers des réactions développées dans un contact quotidien et de long terme avec les chercheurs. Outre la possibilité d'assister à des événements, l'implication de l'enquêteur sur le long terme permet $\mathrm{d}$ 'avoir accès à des "pratiques non officielles », qui n'apparaissent pas normalement dans les questionnaires, à des faits exceptionnels révélant des "phénomènes latents » jusque-là ignorés.

De plus, selon Lemonnier (2004), après la curiosité initiale provoquée par son arrivée, la durée de présence et d'interaction de l'anthropologue avec les populations locales atténue de façon perceptible l'impact qu'il a sur la société étudiée. De la Soudière (1988) va même plus loin lorsqu'il affirme que le sentiment de "perturber » est surtout le fruit d'une surestimation de son rôle négatif par le chercheur qui compense ainsi son sentiment d'absence de rôle dans la société étudiée. On constate la même impression de "perturber » lors des terrains de courte durée.

Quelle place, donc, est laissée à la possibilité de s'impliquer sur un terrain pour une durée limitée? La réponse dépend de la manière dont la recherche est pratiquée, par qui, et avec quels objectifs. Certaines ONG environnementalistes manquent certes de fonds, de temps et d'expertise pour faire correctement leur travail (Suharno et Friedberg, 2003), mais elles sont aussi, et avant tout, portées par des actions cherchant à avoir un impact sur le court terme, plutôt que par la volonté de trouver des solutions durables qui répondent aux besoins des populations locales. Des agents du développement ou de la conservation peuvent aussi pécher par une fausse représentation des réalités d'une société, ou de la réalité que cette dernière veut bien montrer (Novellino, 2003). Mais d'autres utilisent un savoir-faire et un code éthique qui leur permettent de travailler de manière plus indépendante, de tenir compte en connaissance de cause des réalités complexes de chaque terrain (Colchester, 2003) et des droits des populations locales à utiliser et contrôler leurs territoires traditionnels (Colchester, 2000). Des éléments supplémentaires de discussion sont le consentement des populations, l'anonymat des informateurs, ou la légitimité des chercheurs dans leurs actions sur les sites d'études et dans les débats sur les relations plus équitables de partage des connaissances et de compensations qui doivent s'établir avec les communautés locales (Alexiades et Laird, 2002).

Dans le cas de populations moins isolées, plus fragiles car soumises à des pressions extérieures plus intensives, l'arrivée de chercheurs l'un après l'autre, de manière quasi ininterrompue, avec le même cortège de questions, marque davantage ces communautés. Elles demandent de plus en plus des comptes sur les objectifs de ces questionnements, sur l'usage des résultats et sur les droits de propriété intellectuelle sur les données (Posey et al., 1995). Les communautés s'interrogent aussi sur la publication ou non des résultats par le chercheur. Dans le cadre du MLA, toutes les informations reçues ne sont pas enregistrées, comme la préparation des médicaments 
traditionnels, par exemple, et toutes les données récoltées ne sont pas rendues publiques (Sheil et al., 2004). Les cartes participatives, considérées comme leur propriété, ainsi que toute autre information recueillie sur le terrain, sont restituées aux populations (Shanley et Laird, 2002 ; Sheil et al., 2004). Tout usage ultérieur, notamment pour informer les institutions et autres décideurs de la richesse des savoirs locaux, doit passer par l'approbation préalable des principaux intéressés. Les cartes ne sont jamais utilisées dans leur intégralité pour les publications et les données sensibles en sont supprimées (ressources précieuses, lieux sacrés, etc.). Les échantillons d'herbier sont traités sur place, par des laboratoires nationaux, aucun spécimen vivant n'est collecté.

Intégrer les perceptions et les savoirs locaux dans un processus de décisions pose des problèmes d'éthique et de différences culturelles, de perception et d'intérêt des populations locales pour le maintien des ressources naturelles. Selon Laird et Posey (2002), les codes d'éthique et les lignes directrices de recherche sont des éléments importants de tout travail de terrain, en particulier pour les recherches sur la biodiversité qui intègrent des aspects pluridisciplinaires où les droits des populations locales doivent être reconnus (Colchester, 2000).

Hart (2002) propose quatre modèles ou options que suivent en général les anthropologues travaillant sur le développement : apporter de l'information sur les communautés locales à la bureaucratie; prendre parti pour ces communautés pour les aider à défendre leurs intérêts ; essayer de jouer le rôle de médiateur entre les différents intérêts en jeu; ou participer plus directement au "développement ", en essayant de changer la situation de l'intérieur. De nombreux chercheurs deviennent souvent les intermédiaires entre populations locales et décideurs, parfois par choix, parfois par la force des choses. L'approche du MLA permet également de jouer ce rôle dans les rapports entre les différents acteurs de la gestion d'un territoire, en apportant des informations qui permettent de mieux intégrer les perceptions et les priorités locales dans les processus de décisions.

\section{Évaluer l'importance du paysage et des ressources pour les communautés locales}

Étudier les perceptions locales permet de mieux comprendre les priorités en matière de gestion de l'environnement, à l'échelle d'une communauté (Sheil et al., 2004).

Par perceptions locales, nous entendons la manière dont les communautés se représentent leur environnement au regard de leur économie, de leurs activités, de leurs rituels, de leur organisation sociale, du système foncier et de leur cosmogonie. Ces perceptions sont en état de construction permanente, elles n'ont pas un caractère statique, mais dépendent plutôt des transformations propres à chaque société et des contacts établis avec des agents extérieurs - ONG, institutions gouvernementales, voire la communauté scientifique.

Tous ces éléments, qui permettent de décrire une société donnée, nous informent également sur sa vision de la nature et sa place dans le monde. La figure 1 représente une carte des ressources naturelles et des composantes du paysage, établie, dès le début et tout au long d'un terrain de MLA, avec la participation d'une communauté de la région du fleuve Mamberamo, en Papouasie occidentale (ex-Irian Jaya). Cette carte, dessinée par les villageois à partir d'un fond où figuraient les principaux éléments du paysage (villages, rivières principales), a permis d'établir un langage commun entre ces communautés et l'équipe de scientifiques venus travailler avec elles. Elle a permis aux villageois de discuter entre eux de la richesse de leur environnement; mais surtout, cette vision commune de leur territoire apporte un outil de négociation précieux vis-à-vis des institutions locales et des entreprises essayant d'exploiter les ressources naturelles. Prise comme un outil et non comme une fin en soi, cette carte mentale, «dessinant les représentations qu'ont les usagers des espaces proches et lointains, frontières subjectives que ceux-ci assignent à leur région » (Bromberger, 1984), permet de collecter un nombre important d'informations sur les perceptions locales et sur les composantes du paysage (noms et places de toutes les rivières du territoire, emplacement des principales ressources, des montagnes, des différents types de végétation, des anciens villages, y compris des forêts sacrées, hantées ou taboues). De plus, assister aux discussions qui accompagnent son élaboration nous permet de mieux connaître la manière dont ces savoirs et ces perceptions sont partagés au sein de la société. Les informations tirées de cette carte seront utilisées pour tout le reste du travail, notamment pour le choix des sites où sont effectués des relevés de parcelles en forêt. Cet exercice de cartographie est en général réalisé par plusieurs groupes représentatifs du village : par exemple, les jeunes et les adultes, les femmes et les hommes, ou des groupes représentant une activité professionnelle (instituteur, infirmier, chasseurs, pêcheurs) ou traditionnelle (guérisseur, chef coutumier, personnages importants du village). Toutes les cartes dessinées sont réunies en une carte commune, que chacun pourra compléter durant le séjour et qui sera laissée aux villageois à la fin ${ }^{4}$.

\footnotetext{
${ }^{4}$ Cette carte est donc l'association de trois ou quatre cartes réalisées par les différents groupes de villageois, les connaissances et les représentations de chaque groupe pouvant différer. Le fait de les rassembler en une seule carte n'apporte pas une information unique, mais plutôt commune des connaissances de la société sur les paysages et les ressources. De plus, les discussions entre villageois autour de la réalisation de chaque carte sont également documentées.
} 


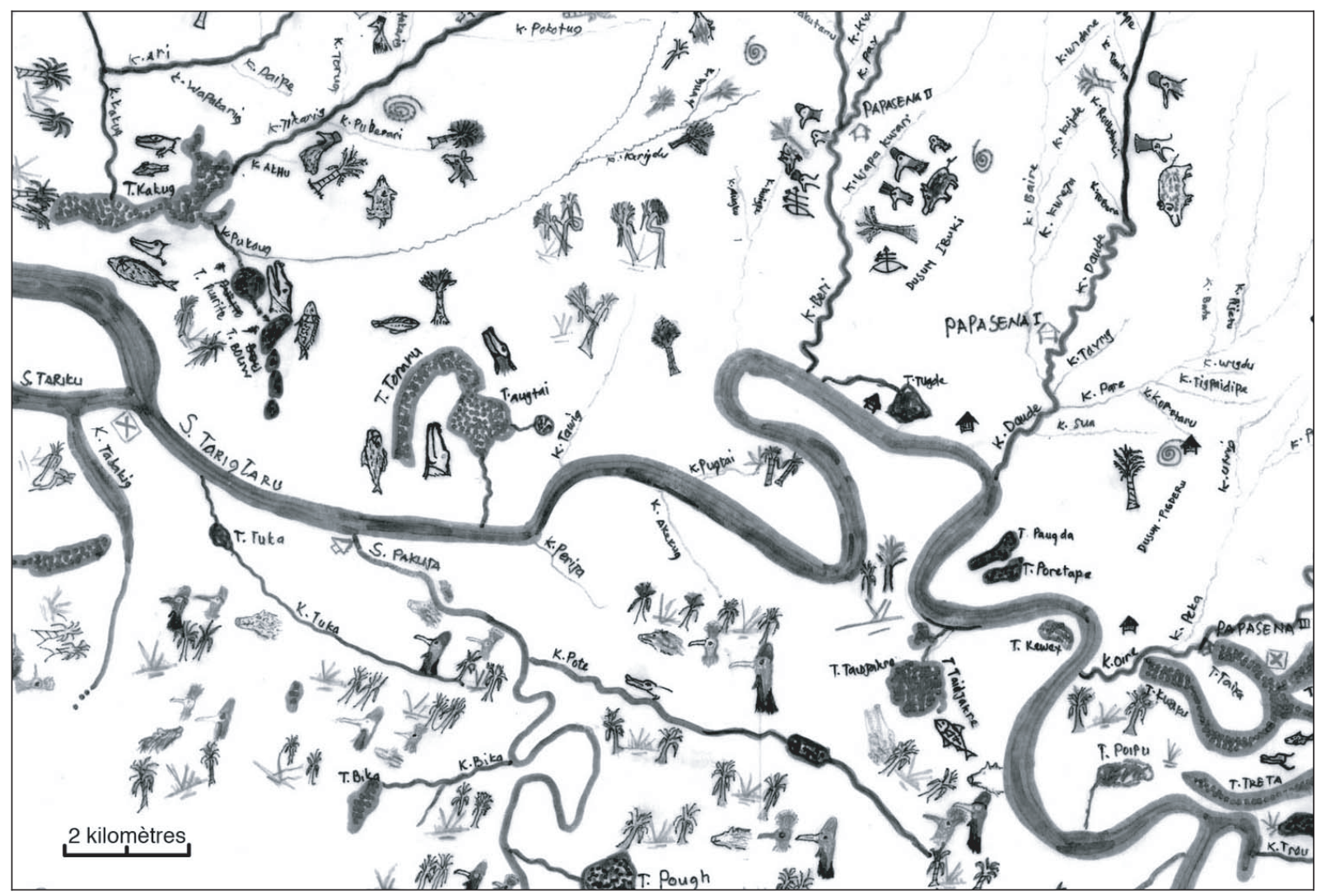

Fig. 1. Carte participative des ressources réalisée par des habitants du bassin de la rivière Mamberamo (Papouasie occidentale, Indonésie). (Source : CIFOR, 2004.)

L'étude est effectuée par deux équipes : l'une, travaillant dans le village; l'autre, à l'extérieur, en forêt $t^{5}$. L'équipe du village travaille sur les relations de la population avec son environnement, sur ses perspectives et sur ses souhaits pour la gestion des ressources et le développement du village. Des outils tels que les réunions communautaires, les questionnaires par foyer, l'observation participative, les enquêtes semi-directives et les exercices d'évaluation en groupe sont utilisés. Des informations sont ainsi recueillies sur la démographie, l'éducation, les sources principales de revenus, l'histoire du village, les différentes unités paysagères identifiées par les villageois, mais aussi les risques et dangers qui menacent le village et les ressources alentour, menaces pour le mode de vie local et pour les ressources naturelles.

Des exercices d'évaluation, ou MDC («méthode de distribution de cailloux»), permettent de recueillir des informations sur l'importance relative des types de paysage en fonction des activités menées (chasse, collecte de bois de feu, de plantes médicinales, etc.), sur l'importance

\footnotetext{
${ }^{5}$ L'équipe «village», de deux à quatre personnes, est composée en général de chercheurs et enquêteurs en sciences sociales, alors que l'équipe de terrain inclut un ou deux botanistes, un ethnobotaniste, un pédologue et leurs assistants et informateurs.
}

de la forêt d'un point de vue diachronique, sur l'importance relative des différentes ressources sauvages ou domestiques et celle des différentes espèces de plantes ou d'animaux. Cette méthode consiste à distribuer cent cailloux entre les différents objets, en fonction de l'importance relative que les villageois leur accordent (Photo). Le résultat doit être commenté et expliqué par les participants après chaque exercice. Ces exercices sont faits en groupes, de la même façon que la cartographie participative.

L'équipe de terrain effectue des relevés de parcelles, choisies sur la base des informations données par l'équipe de village : au moins une parcelle est étudiée dans chaque type de végétation ou lieu d'importance particulière, parfois plus si la diversité est grande. Pour chaque parcelle, des informations sont collectées concernant l'histoire de son emplacement, les noms et usages des plantes, la faune que l'on y trouve, les différents types de sol et leur fertilité relative d'après les informateurs. Des herbiers sont alors constitués qui, après identification, formeront la base de notre analyse.

Soin est pris d'identifier des informateurs, dont certains sont désignés par les villageois, pour les données nécessitant des connaissances spécifiques, telles que l'histoire du village ou bien l'utilisation des plantes, des animaux et des sols. 


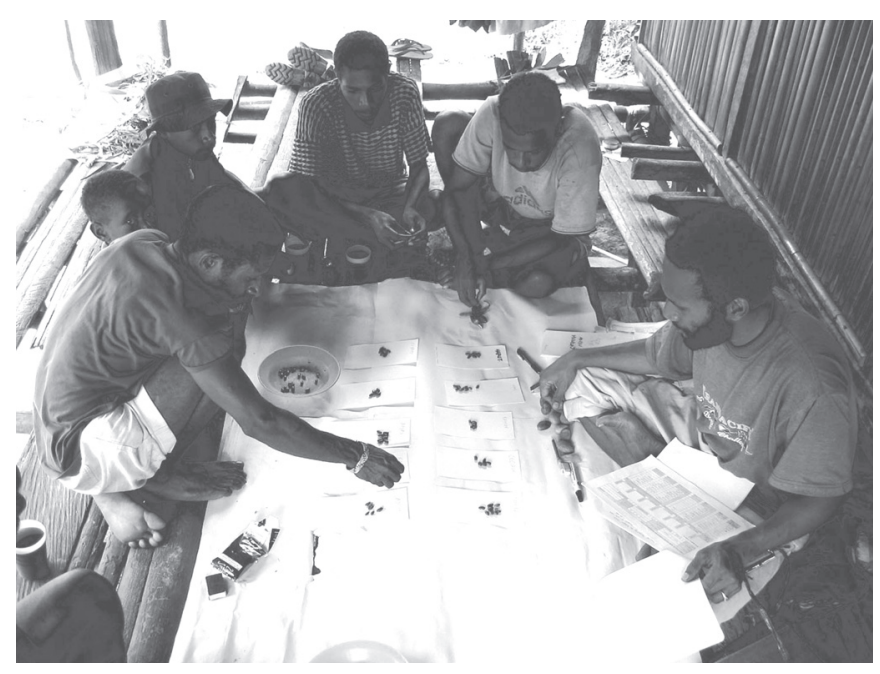

Photo. Exemple d'exercice d'évaluation (ou méthode de distribution des cailloux) dans le bassin de la rivière Mamberamo (Papouasie occidentale). (Photo : M. Boissière.)

Les informations relevées dans le village et en forêt sont comparées chaque jour et d'éventuelles contradictions vérifiées lors des jours suivants. La progression dans la compréhension des relations de la société avec son milieu naturel, et de l'importance de ce dernier pour les activités quotidiennes, se fait en commun, à mesure que les difficultés sont surmontées et après des discussions quotidiennes entre les membres de chaque équipe.

Le choix des sites dépend des différents projets dans lesquels ce jeu de méthodes a été utilisé. Le premier terrain, à Kalimantan, a été choisi pour mettre au point la méthodologie, sur un site où le CIFOR avait déjà de nombreuses activités. Les terrains suivants (par exemple au Cameroun, Gabon, Vietnam) ont été choisis en fonction des opportunités de projets et pour tester le MLA dans différentes situations (populations rurales, forestières, plus ou moins isolées). L'étape suivante, actuelle, est d'utiliser le jeu de méthodes pour répondre à un problème ou un enjeu particulier, posé par une institution (par exemple, Conservation International en Papouasie occidentale) ou un projet (Union européenne aux Philippines). Cette étape suppose des adaptations des méthodes aux questionnements de départ, auxquelles chaque chercheur membre de l'équipe prend part. En ce sens, l'approche diffère considérablement, une fois de plus, de celle des ONG et des agents du développement, qui utilisent des outils (RAP/RRA/PRA) systématiquement, sans véritable souci de les adapter aux réalités du terrain. Nous donnons ci-dessous quelques exemples de résultats obtenus avec des applications possibles à des problèmes de conservation, de gestion durable des écosystèmes forestiers et d'implication des populations locales dans la gestion des ressources.

\section{Le sagou à Kalimantan Est (Indonésie)}

Lorsqu'ils sont loin de leur village, les Punan du bassin de la rivière Malinau, dépendent des ressources forestières pour leur subsistance, notamment des sagoutiers sauvages. En forêt primaire, cette espèce de palmier est fréquemment récoltée et donc protégée par les villageois. Mais dans les forêts soumises à l'exploitation forestière, les sagoutiers deviennent rares. L'espèce principale, Eugeissona utilis, croît en général sur les crêtes (Meijaard et al., 2005), lieu de passage des routes forestières pour les exploitations à impact réduit (Reduced Impact Logging [RIL]). Ces techniques d'exploitation, reconnues comme plus respectueuses de l'environnement, détruisent en réalité les ressources en sagou sauvage des Punan. Ces pratiques forestières devraient davantage prendre en compte leurs effets sur les modes de vie des populations locales (Sheil et al., 2006).

\section{Désengagement des communautés locales au Vietnam}

Dans le village de Khe Tran, au centre du Vietnam, et au bord de la future réserve naturelle de Phong Dien, les villageois appartenant au groupe des Pahy pratiquaient jadis une agriculture rotative sur brûlis. Depuis une dizaine d'années, ils sont encouragés par le gouvernement à se tourner vers des activités agricoles sédentaires qui sont devenues des activités lucratives importantes pour cette société (Boissière et al., 2006). Toute activité agricole et extractiviste est interdite dans les limites de la future aire protégée (Birdlife International, Forest Inventory and Planning Institute, 2001). Il s'ensuit un désengagement des populations locales vis-à-vis des activités dans la forêt, une perte des savoirs liés aux ressources naturelles. Dorénavant, l'usage de plantes sauvages le plus fréquent, identifié par les villageois, est pour nourrir les buffles. À mesure que les savoirs sur les ressources naturelles se perdent, l'intérêt pour le territoire compris dans l'aire protégée diminue. Cet exemple plaide en faveur d'une évolution nécessaire de la perception des institutions de conservation à leur égard.

\section{Conservation avec les populations locales en Papouasie occidentale}

Le MLA a aidé une importante ONG pour la conservation de la nature à bâtir une aire protégée dans le bassin du fleuve Mamberamo, en se basant non seulement sur une étude de la biodiversité, mais aussi sur les priorités locales (CIFOR, 2004). Lors de l'étude, des relations prometteuses entre les différentes parties concernées ont été construites, fondées sur une même compréhension de ce qui pourrait et devrait être fait en matière de conservation dans cette région. 
Les situations variées présentées dans ces exemples montrent l'intérêt possible du MLA dans le cadre aussi bien de projets de développement, de conservation que de recherche appliquée.

\section{Intérêts d'une approche intégrée des perceptions}

Un avantage majeur de cette approche est l'intégration qu'elle permet entre informations venant des sciences humaines et des sciences naturelles. Certains questionnements propres aux sciences humaines (sur l'importance des composantes du paysage) trouvent des réponses dans le travail de terrain (typologie des paysages, caractérisation des forêts et importance des savoirs sur les ressources naturelles). Ainsi, les données de terrain ne sont plus séparées les unes des autres, indépendantes et parfois même en contradiction, mais chacune est étroitement connectée aux autres. Par exemple, au Gabon, les composantes du paysage (types de forêts) les plus importantes pour les communautés du Parc national de l'Ivindo, et selon les MDC, étaient celles qui contenaient la plus grande richesse en espèces et le plus grand pourcentage d'espèces utiles selon l'étude botanique et ethnobotanique (Sassen et Wan, 2006). Cette intégration des résultats permet des études plus fines et plus pertinentes sur la perception locale de l'environnement, ce qui n'est pas le cas des enquêtes «rapides » et superficielles comme les PRA.

Un exemple de cette intégration concerne le choix des sites où l'on effectue des relevés de parcelles. Ces dernières permettent d'abord d'étudier la biodiversité, mais également de préciser les savoirs et les savoir-faire des populations locales, et de caractériser les paysages en fonction de ceux-ci. Il est donc important d'identifier les composantes du paysage selon le point de vue local, mais aussi de décider, pour chacune d'elles, en coordination avec les villageois, quels sont les sites les plus représentatifs. La cartographie participative permet cette intégration, en complément de questionnaires portant sur les ressources, les paysages et l'histoire du village.

La MDC est un élément important des méthodes utilisées, car elle permet de collecter mais aussi de quantifier de manière relative les perceptions locales sur les paysages et les ressources forestières. Toutefois, plus que de simples méthodes quantitatives, ces exercices encouragent les communautés locales à exprimer ce qui, selon elles, est important et pourquoi. Une valeur est ainsi donnée à des ressources ou des paysages dont l'importance était souvent sous-estimée et parfois même ignorée par les décideurs (Sheil et Liswanti, 2006), ainsi que nous le montre l'exemple des zones à sagoutiers de Kalimantan.

Ce jeu de méthodes nous permet d'avoir une image générale des relations des communautés locales avec leur environnement naturel et des menaces qui pèsent sur cette relation. Les informations produites permettent d'obtenir un premier aperçu, et ainsi de prévoir des activités à mener sur un plus long terme, en fonction de la discipline et des objectifs (anthropologie, écologie, conservation, développement durable des communautés, etc.). Cependant, la qualité de l'information dépend étroitement de la manière dont le chercheur utilise les méthodes, et surtout du contexte local. Ce dernier peut être limitant en raison de problèmes d'ordre politique (guérilla en Papouasie occidentale, méfiance institutionnelle au Vietnam), social (populations marginales aux Philippines, problèmes d'alcoolisme et de violence pour certaines sociétés du Gabon, etc.) et logistique (sites d'accès difficiles en Papouasie occidentale, formalités administratives complexes pour l'obtention d'autorisations au Vietnam).

Le MLA ne se veut pas enfermé dans un carcan de méthodes dont on ne peut sortir et dont on doit respecter toutes les étapes scrupuleusement pour garantir le bon résultat, comme les kits proposées par la FAO (1990) pour les programmes de foresterie communautaire. Ce jeu de méthodes se veut au contraire flexible et surtout adaptable au contexte local et au questionnement de départ.

\section{Problèmes et précautions d'usage pour cette approche ou l'art de marcher sur des œufs}

Les problèmes et les difficultés que l'on peut rencontrer en utilisant cette approche sont abordés dans l'ouvrage de Sheil et al. (2004), mais nous souhaitons ici préciser certains aspects qui nous paraissent particulièrement importants.

Le principal problème lié à l'utilisation de ce type de méthode pluridisciplinaire est dû au langage et à la difficulté d'établir des définitions communes de concepts de base avec les communautés locales, mais aussi au sein de l'équipe de chercheurs.

Les notions de "paysage ", de "foyer ${ }^{6}$ ", mais aussi le concept de «conservation de la biodiversité » ou le terme de «forêt », font rarement écho à des perceptions et une nomenclature locales ou alors sont perçus de manière totalement différente entre chercheurs et communautés, voire entre les chercheurs de différentes disciplines.

Ces difficultés ne sont pas propres au MLA et se retrouvent dans de nombreuses recherches sur les savoirs locaux, mais la durée limitée des terrains et le fait que la plupart des activités sont basées sur l'hypothèse d'une définition et d'une vision communes d'importants concepts de base, rendent la tâche plus difficile.

\footnotetext{
6 «Foyer» est ici pris dans le sens de plus petite unité familiale. Les éléments que couvre ce terme peuvent cependant varier en fonction des interprétations.
} 
Le manque de maitrise de la langue locale accentue le problème du partage de mêmes définitions et de l'appréhension de certains concepts locaux concernant la perception et l'usage des paysages. Aux interprétations des propos d'informateurs et aux énoncés théoriques par les chercheurs en sciences sociales, il est souvent nécessaire d'ajouter une traduction, qui est alors source de nouvelles interprétations (Olivier de Sardan, 1998). Chez les Batak de l'île de Palawan (Philippines), par exemple, de nombreuses enquêtes ont été nécessaires pour définir les principales unités composant le paysage. Ne parlant ni le tagalog (langue nationale) ni le batak, nous étions obligés de passer par des interprètes, ce qui introduisait un biais important dans les réponses.

Un premier cycle d'entretiens en groupe a permis d'établir les noms des principaux types de forêts selon les Batak, aussitôt déniés par des entretiens avec les shamans lors de nos sorties en forêt. D'autres discussions, avec différents groupes, ont permis de mieux définir les questions et par conséquent d'obtenir des réponses plus justes. Définir correctement les composantes du paysage identifiées par les villageois est important, car elles servent de base pour l'application de la MDC.

L'usage d'interprètes crée également une barrière entre les villageois et les chercheurs, ce qui limite la capacité à développer des relations de confiance. L'impact sur la qualité des informations récoltées peut être assez important. Lors des différentes études pour lesquelles le MLA a été utilisé, le problème de la langue, mais aussi la nature très académique des recherches menées ont donné lieu à des difficultés pour expliquer aux communautés villageoises nos activités et nos méthodes. Les villageois ont souvent du mal à imaginer de quelle façon notre étude et ses résultats peuvent leur être utiles dans la vie quotidienne et dans leurs rapports avec les institutions gouvernementales. Dans certaines régions, les communautés ont vu passer déjà beaucoup de chercheurs ou d'ONG aux intérêts divers. Ces expériences, souvent mal vécues ou perçues comme improductives par les communautés, les ont rendues méfiantes envers les étrangers.

De nombreux facteurs locaux peuvent affecter la qualité des réponses qui nous sont faites, lors des exercices de MDC ou lors des questionnaires. Des stratégies locales influencent souvent et déforment ces réponses, qui peuvent être "de complaisance», ainsi que l'explique Céfaï (2003) : c'est le cas, par exemple, des luttes de pouvoir, des conflits avec d'autres communautés, du besoin de reconnaissance d'individus au sein du village et de la visibilité que donne aux acteurs la collaboration avec le projet.

En dehors de ces aspects politiques, les réponses peuvent aussi être influencées par la présence, dans le passé, d'autres projets porteurs de concepts plus ou moins bien intégrés par les populations locales et de discours qui se reflètent dans le positionnement des villageois par rapport à nos activités et dans la qualité des informations reçues.

Les exercices d'évaluation tels que ceux de la MDC ne devraient être utilisés que dans le contexte d'une recherche pluridisciplinaire, comme celle du MLA, où l'interaction des différentes activités leur donne toute leur légitimité. La génération de chiffres, traduisibles en pourcentages, peut suggérer une précision mathématique qui n'est pas forcément l'expression de la réalité, même si, selon Sheil et Liswanti (2006), elle s'en rapproche sans doute davantage que des considérations uniquement qualitatives. Ces chiffres doivent être utilisés de manière relative, en les comparant entre eux, et les extrêmes apportent souvent plus d'informations que les chiffres en soi : par exemple, il y a peu de différence entre 13 ou 14 cailloux attribués à deux catégories de paysage pour un usage donné, mais une valeur de 50 donnée à la forêt sera visible si on la compare aux 5 cailloux donnés, par exemple, aux jardins. Les explications et les discussions après chaque exercice permettent de mieux comprendre les facteurs locaux influant les perceptions et donc les réponses. Cela étant, les interprétations sont nombreuses, certains individus prennent un ascendant sur les autres, et les chiffres peuvent changer si le même exercice est répété avec le même groupe. Le type de réponses obtenues dépend de la composition des groupes (selon le sexe, l'âge, les professions, etc., des participants) et ne représente pas forcément l'ensemble de la communauté.

De manière plus générale, le MLA produit une quantité importante de données à saisir, à traiter, et à analyser, et cela demande du temps, comparativement à la durée de chaque terrain. Des adaptations de ce jeu de méthodes aux diverses problématiques peuvent réduire cette quantité de données, en se concentrant sur certains aspects seulement (par exemple, mieux cibler les MDC, réduire le nombre de parcelles, ne pas étudier les sols). Cependant, l'ensemble des méthodes permet une étude pluridisciplinaire et intégrative de plusieurs points de vue : celui des populations locales en priorité, mais décliné par les diverses disciplines représentées dans l'équipe. Enlever ces diverses entrées disciplinaires pour ne garder qu'un petit nombre d'activités risque de retirer de la valeur aux informations collectées.

\section{Conclusion}

Le jeu de méthodes développé par le CIFOR fournit des informations sur le point de vue local sur la gestion des paysages et des ressources forestières, sur les systèmes de perception de l'environnement et sur les priorités de communautés dépendant de la forêt et des produits forestiers pour leurs activités quotidiennes. Ce sont des méthodes de recherche essentiellement qualitatives et assez générales, qui peuvent être prises comme 
base pour élaborer des recherches plus approfondies en écologie (dynamiques forestières en fonction des activités humaines), en anthropologie (systèmes de représentation du monde par les communautés locales, problèmes liés au foncier) et dans de nombreux autres champs disciplinaires. Elles permettent d'estimer plus facilement si ces divers aspects sont indispensables (ou non) pour comprendre la façon dont les populations rurales gèrent leurs relations avec l'environnement et l'importance qu'ont pour elles les ressources forestières. Dans l'exemple du Vietnam, elles permettent de s'affranchir du stéréotype de populations forestières figées dans leurs savoirs traditionnels et leurs perceptions. Leur refuser toute activité au sein d'une aire protégée, c'est courir le risque de voir disparaître ces savoirs et de perdre un allié précieux pour la gestion durable de ces espaces (Boissière et al., 2006).

Le chercheur utilisant ce jeu de méthodes se heurte aux difficultés propres aux études fondées sur un intense travail de terrain dans une durée limitée, au contact de populations locales qui ont leur propre agenda politique ou leurs propres conceptions de ce que doit être le développement à l'échelle locale. Il peut alors devenir l'otage de conflits internes à la société étudiée, liés aux luttes de pouvoir, par exemple, ou aux problèmes fonciers. Il dépend aussi de ses propres préjugés, des différences culturelles et de la pluralité des perceptions au sein de son équipe. Ces difficultés sont autant d'embûches à surmonter pour rendre compte le plus honnêtement possible de la réalité d'une situation, en suivant une éthique la plus rigoureuse possible. S'agit-il d'une mission impossible? Les longues études de terrain, avec un investissement du chercheur sur le long terme, sont-elles les seules façons correctes de collecter, partager et restituer des connaissances, ainsi que le suggère Céfaï (2003) ? On peut imaginer, au contraire, un point de rencontre entre ces deux visions opposées de la recherche, une complémentarité avec, d'un côté, la mise en évidence de sujets sensibles, d'éléments caractéristiques d'une situation étudiée dans son contexte, et, de l'autre, l'approfondissement d'aspects davantage liés à une discipline. Olivier de Sardan (1995) critique avec raison les études bâclées, menées par des scientifiques mal formés avec des "méthodologies clés en main », et s'oppose ainsi aux méthodes rapides du genre des PRA. Il s'oppose également à l'usage systématique d'entretiens collectifs comme source d'information valable. Il reconnaît cependant la difficile compatibilité entre de très longs terrains de recherche académique, portés par des «problématiques abstraites », et la demande des institutions de développement et des décideurs. Il propose comme solution de recourir le plus souvent possible à des spécialistes d'une région ou d'un thème pour les «expertises » envisagées, et de combiner enquêtes individuelles et collectives, de court et moyen terme, pour augmenter la validité des résultats. Le MLA semble permettre cela et ouvrir la porte à plus de collaboration interdisciplinaire et institutionnelle. Le jeu de méthodes est mis en œuvre par des spécialistes des disciplines utilisées. Il doit rester un outil flexible, adaptable aux diverses problématiques de conservation et de développement. Il ne s'agit pas d'une « recette», mais plutôt d'un cadre de travail pour des scientifiques ayant une bonne expérience de terrain, avec la possibilité d'adapter ce cadre aux différents sites, contextes et projets. La quantité d'informations produites ne donne pas des réponses directes aux problèmes de développement et de gestion des ressources naturelles, mais permet plutôt d'explorer les points d'intérêts communs entre les différents acteurs du développement, en y intégrant celui, fondamental, des populations locales. Considéré comme un premier pas pour une meilleure compréhension des rapports complexes entre sociétés forestières et environnement, cet outil est susceptible de dégager des opportunités et d'engager une discussion entre les différents acteurs concernant la gestion de la biodiversité.

\section{Références}

Alexiades, M., Laird., S., 2002. Laying the foundation: equitable biodiversity research relationships, in Laird, S. (Ed.), Biodiversity and Traditional Knowledge, London, Earthscan, 3-15.

Birdlife International, Forest Inventory and Planning Institute (Publ.), 2001. Phong Dien proposed Nature Reserve, in Sourcebook of Existing and Proposed Protected Areas in Vietnam, Hanoi.

Boissière, M., Sassen, M. 2005. Perceptions of local perception... Relevance and difficulties of assessing the local importance of natural resources using a multidisciplinary approach. Communication présentée au IV ${ }^{e}$ Congrès international d'ethnobotanique (ICEB 2005), Istanbul, Yeditepe University, 21-26 août.

Boissière, M., Basuki, I., Koponen, P., Wan, M., Sheil., D., 2006. Biodiversity and Local Perceptions on the Edge of a Conservation Area, Khe Tran village, Vietnam, Bogor, CIFOR.

Bromberger, C., 1984. Des cartes ethnologiques : pourquoi faire?, Terrain, 3 (http://terrain.revues.org/document2819.html).

Céfaï, D. (Ed.), 2003. L'Enquête de terrain, Paris, La Découverte/MAUSS.

Chambers, R., 1994 [ $1^{\text {re }}$ éd. 1993]. Challenging the Professions: Frontiers for Rural Development, Bath, Intermediate Technology Publications.

CIFOR, 2004. Building Capacity for Multidisciplinary Landscape Assessment in Papua: Three Phases of Training and Pilot Assessments in the Mamberamo Basin. Report for Conservation International (Papua Program), Bogor.

Colchester, M., 2000. Self-determination or environmental determinism for indigenous peoples in tropical forest conservation, Conservation Biology, 14, 5, 1365-1367.

Colchester, M., 2003 [1 $1{ }^{\text {re }}$ éd. 1994]. Salvaging Nature: Indigenous Peoples, Protected Areas and Biodiversity Conservation, Genève, World Rainforest Movement, Forest Peoples Programme, WWF, United Nations Research Institute for Social Development. 
Conway, G.R., 1987. Agroecosystem Analysis for Research and Development, Bangkok, Winrock International.

Cyranoski, D., 2006. Calls to conserve biodiversity hotspots, Nature, 439, 774.

FAO, 1990 (Publ.). The Community's Toolbox: The Idea, Methods and Tools for Participatory Assessment, Monitoring and Evaluation in Community Forestry, Rome.

Friedberg, C., 2006. Compte rendu de Douglas Sheil et al., À la découverte de la biodiversité de l'environnement et des perspectives des populations locales dans les paysages forestiers, Natures Sciences Sociétés, 14, 1, 104-106.

Hart, K., 2002. Quelques confidences sur l'anthropologie du développement, ethnographiques.org, 2 (http://www.ethnographiques.org/2002/Hart.html).

Holland, J., Blackburn, J. (Eds), 1998. Whose Voice? Participatory Research and Policy Change, Bath, Intermediate Technology Publications.

IFAD, ANGOC, IIRR (Publ.), 2001. Enhancing Ownership and Sustainability: A Resource Book on Participation, Manila.

Laird, S., Posey, D.A., 2002. Professional society standards for biodiversity research: codes of ethics and research guidelines, in Laird, S. (Ed.), Biodiversity and Traditional Knowledge, London, Earthscan, 16-38.

Latour, B., 2001. Le Métier de chercheur : regard d'un anthropologue, Paris, INRA Éditions.

Lemonnier, P., 2004. Sous le regard de saint Pataugas : à propos d'âge de pierre, de globalisation et de responsabilité de l'ethnologue, ethnographiques.org, 5 (http://www.ethnographiques.org/2004/Lemonnier.html).

McKenna, S.A., Allen, G.R., Suryadi, S. (Eds), 2002. A marine rapid assessment of the Raja Ampat Islands, Papua Province, Indonesia, RAP Bulletin of Biological Assessment, 22.

Meijaard, E., Sheil, D., Nasi, R., Augeri, D., Rosenbaum, B., Iskandar, D., Setyawati, T., Lammertink, M., Rachmatika, I., Wong, A., Soehartono, T., Stanley, S., O’Brien, T., 2005. Life after Logging: Reconciling Wildlife Conservation and Production Forestry in Indonesian Borneo, Bogor, CIFOR.

Novellino, D., 2003. From seduction to miscommunication: the confession and presentation of local knowledge in 'participatory development', in Pottier, J., Bicker, A., Sillitoe, P. (Eds.), Negotiating Local Knowledge: Power and Identity in Development, London, Pluto Press.

Olivier de Sardan, J.-P., 1995. Anthropologie et développement : essai en socio-anthropologie du changement social, Paris, APAD/Karthala.

Olivier de Sardan, J.-P., 1998. Émique, L'Homme, 147, 151166 (http://www.persee.fr/showPage.do?urn=hom_04394216_1998_num_38_147_370510).
Posey, D., Dutfield, G., Plenderleith, K., 1995. Collaborative research and intellectual property rights, Biodiversity and Conservation, 4, 892-902.

Richards, S.J., Suryadi, S. (Eds.), 2002. A biodiversity assessment of Yongsu-Cyclops Mountains and the Southern Mamberamo Basin, Papua, Indonesia, RAP Bulletin of Biological Assessment, 25.

Sassen, M., Wan, M., 2006. Biodiversité et priorités locales dans une communauté riveraine du Parc national de l'Ivindo, Gabon. Rapport d'une mission de recherche dans le cadre du projet Réhabilitation et re-dynamisation de la station de recherche de l'IRET à Makokou/Ipassa, IRET/CENAREST/CIFOR, Bogor, CIFOR.

Schwartz, O., 1993. L'empirisme irréductible, in Anderson, N., Le Hobo : sociologie du sans-abri, Paris, Nathan, 265-305.

Selener, D., 1997. Participatory Action Research and Social Change, New York, Cornell University.

Shanley, P., Laird, S., 2002. 'Giving back': making research results relevant to local groups and conservation, in Laird, S. (Ed), Biodiversity and Traditional Knowledge, London, Earthscan, 102-124.

Sheil, D., Wunder, S., 2002. The value of tropical forest to local communities: complications, caveats, and cautions, Conservation Ecology, 6, 2 (http://www.consecol.org/vol6/iss2/art9).

Sheil, D., Puri, R.K., Basuki, I., Van Heist, M., Syaefuddin, Rukmiyati, Agung Sardjono, M., Samsoedin, I., Sidiyasa, K., Chrisandini, Permana, E., Mangopo Angi, E., Gatzweiler, F., Johnson, B., Wijaya, A., 2004. A la découverte de la biodiversité, de l'environnement et des perspectives des populations locales dans les paysages forestiers: méthodes pour une étude pluridisciplinaire du paysage, Bogor, CIFOR

(http://www.cifor.cgiar.org/mla/_ref/fr/publication/ exploring_biological_diversity.htm).

Sheil, D., Liswanti, N., 2006. Scoring the importance of tropical forest landscapes with local people: patterns and insights, Environmental Management, 38, 1, 126-136.

Sheil, D., Puri, R.K., Wan, M., Basuki, I., Van Heist, M., Liswanti, N., Rukmiyati, Rachmatika, I., Samsoedin, I., 2006. Recognizing local people's priorities for tropical forest biodiversity, Ambio, 35, 1, 17-24.

Soudière, M. de la, 1988. "L'inconfort du terrain ", Terrain, 11 (http://terrain.revues.org/document3316.html).

Suharno, D.M.W., Friedberg, C., 2003. Resource management issues: NGOs relate to the new legal framework for local autonomy in Indonesia, International Social Science Journal, $178,573-575$

Reçu le 26 Avril 2006. Accepté le 7 décembre 2006. 